\title{
Dissimilar Behaviour of the Two Kinds of Duplication- Deficiency Female Gametophytes in the T3-6 Interchange Heterozygote of Pearl Millet (Pennisetum glaucum (L.) R. Br.)
}

\author{
K. Uma Devi*, T. V. Rajya Lakshmi, K. Aruna Lakshmi, \\ P. S. R. L. Narasinga Rao and K. Adiseshaiah \\ Department of Botany, Andhra University, Visakhapatnam-530 003, India
}

Accepted January 26, 1998

\begin{abstract}
Summary In structural heterozygotes for the T3-6 chromosomal interchange in pearl millet, the percent pollen fertility correlated with percent seed set. The duplicate-deficient (dp/df) microspores degenerated at binucleate stage. The effect of the $\mathrm{dp} / \mathrm{df}$ condition on the structure and function of the female gametophyte was investigated in this interchange heterozygote. Inflorescences from the interchange heterozygote plants were fixed at five developmental stages:- at pre pollination (i.e. stage 1) and post pollination (i.e. stages 2 to 5) at $24 \mathrm{hr}$ intervals up to $96 \mathrm{hr}$ after pollination (hap). The embryo sac (female gametophyte) was dissected from the ovule and stained with $2 \%$ acetocarmine. In the post pollination samples the rate of early seed development was estimated by counting the number of cells in the embryo and endosperm. A normal looking embryo sac was found in all the ovules at pre pollination stage. Post pollination, two kinds of abnormalities were found in $\sim 50 \%$ of the ovules: some had unfertilized embryo sacs; some showed a lag in development of the embryo and endosperm. These abnormalities could be due to the $\mathrm{dp} / \mathrm{df}$ condition of the embryo sacs - the two kinds representing the two types of $\mathrm{dp} / \mathrm{df}$ products formed in the interchange heterozygote. The difference in tolerance of the $\mathrm{dp} / \mathrm{df}$ condition on the male and female sides is attributed to a) the (programmed) difference in the control of gametophyte development in the two sexes, and b) the existence of competition among the (three) microspore categories (normal, $\mathrm{dp} / \mathrm{df}$ of one kind and $\mathrm{dp} / \mathrm{df}$ of another kind) as opposed to the non-competitive aspect of megaspore development (which ever is the category).
\end{abstract}

Key words Pearl millet (Pennisetum glaucum), Duplication-deficiency spores, Female gametophyte, Embryo sac structure, Post pollination development, T3-6 interchange.

In structural hybrids heterozygous for a chromosomal interchange (reciprocal translocation), nearly half of the meiotic products are genetically imbalanced. The meiocytes in which the interchange multivalent shows an adjacent or indifferent orientation at metaphase produce spores which are duplicate for certain regions of a chromosome and deficient for some regions of another chromosome. These are described as duplication-deficiency (dp/df) spores. Two types of $\mathrm{dp} / \mathrm{df}$ spores are produced from such meiocytes. The chromosomal region which is in duplicate in one kind of dp/df spores is the one for which the other kind is deficient and vice-versa (Fig. 1). The $\mathrm{dp} / \mathrm{df}$ microspores fail to develop into normal male gametophytes (pollen) though they are physically present in a genetically balanced sporophyte. In such microspores, the development of the gametophyte is either not initiated or it is arrested midway leading to the formation of structurally abnormal pollen or, the gametophytes that develop from these microspores look normal but fail to compete with normal ones. The stage at which the development of the male gametophyte is affected depends on the chro-

\footnotetext{
* Corresponding author: Tel. No. 091891574871 Extn. 342. Fax No. 091891555547.
} 

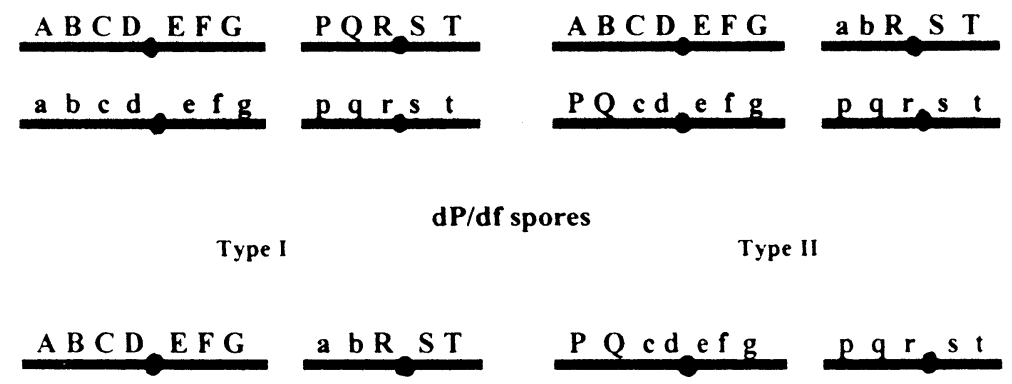

Fig. 1. Chromosome complement (with respect to the chromosomes that were involved in the interchange) of the normal and interchange heterozygote individuals and the $\mathrm{dp} / \mathrm{df}$ meiotic spores.

mosomal region involved. Transmission of deleterious genetic condition to the next generation via the pollen is thus incidentally blocked. This beneficial effect of pollen selection is however diluted due to the absence of such a rigid selection on the female side. Deleterious genotypes and chromosomal aberrations that are occasionally transmitted to the offspring are most often carried through the female gamete (Khush 1973, Sybenga 1992). The extent to which dp/df condition affects female gametophyte development or function in comparison to the male gametophyte has not been analysed in almost all cases of the chromosomal interchanges studied in plants. There is a recent report (Ray et al. 1997) of the study of the female gametophyte in an interchange heterozygote of Arabidopsis thalliana. We have developed a technique of easy dissection of the embryo sac in pearl millet. We investigated the structure and functional ability of the female gametophytes in a structural heterozygote of the well characterized T3-6 interchange (Koduru and Krishna Rao 1984, Uma Devi et al. 1996) of pearl millet.

Approximately $50 \%$ of the microspores in the T3-6 interchange heterozygote of pearl millet degenerate after one pollen mitosis i.e. at the binucleate stage (Fig. 2a) and appear as ghosts in squashes made from mature anthers. These are assumed to be dp/df (Adiseshiah 1995). The percent pollen fertility correlated with the percent seed set in this interchange heterozygote in many genetic backgrounds (Koduru 1979). This indicates that dp/df products are formed in the same frequency on the male and female sides in the T3-6 interchange heterozygote. At what stage the female gametophyte development/function is affected by $\mathrm{dp} / \mathrm{df}$ condition in the megaspores in this interchange heterozygote was investigated.

\section{Material and methods}

To get a population of plants heterozygous for the T3-6 interchange, a cross was made between the semidwarf T3-6 interchange homozygote line IP12781 and a genic male sterile segregant of a normal disomic inbred line Vg272. Eighty plants from the crossed seed were raised. Five of these plants picked at random possessed interchange multiples in pollen mother cells at diakinesis; the sample of 80 plants is thus taken to be a group of interchange heterozygotes.

To study the structure and functional competence of the female gametophytes in the interchange heterozygote, ears were fixed at five developmental stages. Bagged ears with styles fully emerged and ready for pollination were fixed to represent pre-pollination stage. For post pollination developmental stages, ears were artificially pollinated with fertile pollen from the disomic inbred $\mathrm{Vg} 272$. They were fixed post pollination at $24 \mathrm{hr}$ intervals up to 96 hap to represent four developmental stages at 24, 48, 72 and 96 hap. 
Two ears for each developmental stage were analysed. About 125 ovaries were dissected out from the florets of each ear. The ovary was floated in a drop of water on a slide, the entire embryo sac along with the antipodal cluster was dissected out with fine needles under a stereo dissection microscope with $50 \times$ magnification. The embryo sac was stained with a drop of $2 \%$ acetocarmine and a coverslip applied for examination under a compound microscope.

In the post pollination samples, the rate of early seed development was estimated from ten ovules per sample. The criterion was direct count of the number of cells/nuclei in the embryo and endosperm. In pearl millet, cell (embryo) and nuclear (endosperm) divisions are synchronous up to 48 hap in the embryo and 72 hap in the endosperm. In these tissues up to this time, the number of nuclei/cells therefore double with each round of cell division. An estimate of the number of cell cycles that occurred in the embryo and endosperm in each $24 \mathrm{hr}$ interval after pollination is thus made from the number of nuclei/cells. The mean cycle time for each $24 \mathrm{hr}$ interval after pollination was calculated from the number of cell cycles that occurred within that period. The mean values were compared with the values estimated by Krishna Rao and Aswani Kumari (1985) in the sib crosses of the interchange homozygote (IP12781) line. The rate of early seed development has been studied in more than 15 inbred lines of pearl millet (Aswani Kumari 1981, Krishna Rao and Aswani Kumari 1981, 1985). The rate of development in the T3-6 interchange homozygote (used as control in this study) was similar to the rate in other normal disomic inbreds studied.

Percent pollen fertility and seed set on open pollinated ears of the interchange heterozygote line was calculated in ten plants. To calculate pollen fertility, anthers ready for dehiscence were used.

\section{Results}

In the ears fixed prior to pollination, each of the ovules dissected did have an embryo sac. Visually, (at stereo- and compound microscope level) these embryo sacs were comparable to those in disomic lines (Fig. 2b), they had a well developed egg apparatus with a starch rich egg cell, prominently stained polar nuclei (Fig. 2c, d) and a cluster of antipodal cells. However, seed set in the interchange heterozygote was low. It ranged between 33 to $52 \%$ as is expected from the chromosomal aberration. Therefore, in each of the four post pollination samples collected at $24 \mathrm{hr}$ intervals, the 'embryo sacs' were examined to see if any visual indication(s) of functional inadequancy existed.

Fertilization in pearl millet occurs within an hour after pollination and by 24 hap a two celled embryo and a `64 nucleate endosperm are normal (Krishana Rao and Aswani Kumari 1981). Only 174 of the 250 ovules dissected in the 24 hap samples of the interchange heterozygote showed this level of development (Fig. 2e), the rest of the ovules had unfertilized embryo sacs (Table 1). By 48 hap, normal disomics of pearl millet have a $\sim 18$ celled embryo and $\sim 600$ cells endosperm in most (90-98\%) of the ovules (Krishna Rao and Aswani Kumari 1981, 1985). In the T3-6 interchange heterozygote, of the 250 ovules dissected in the 48 hap sample, only 165 showed the expected number of cells in the embryo and endosperm (Fig. 2f). Unfertilized embryo sacs were found in 40 ovules (Table 1). In 45 ovules, the endosperm was still in the free nucleate stage with a fewer number of nuclei than is expected at this stage; the embryo in these ovules, however, had the expected number of cells (Table 2). The developing seed in normal inbreds of pearl millet at 72 hap is reported to have a $\sim 60$ celled embryo and a $\sim 2900$ celled endosperm and by 96 hap a $\sim 500$ celled embryo and a $\sim 5000$ celled endosperm are found (Krishna Rao and Aswani Kumari 1981, 1985). Only $50 \%$ of the ovules in the 72 and 96 hap samples of the interchange heterozygote showed this level of development (Fig. $2 \mathrm{~g}$, h, Tables 1,2 ). The other $50 \%$ of the ovules in both the samples had either an unfertilized embryo sac or a fewer celled embryo and an endosperm "still in free nucleate condition" with fewer number of nuclei than is expected at these stages of development (Tables 1, 2).

Two kinds of functional disability were thus observed in the ovules from post pollination sam- 


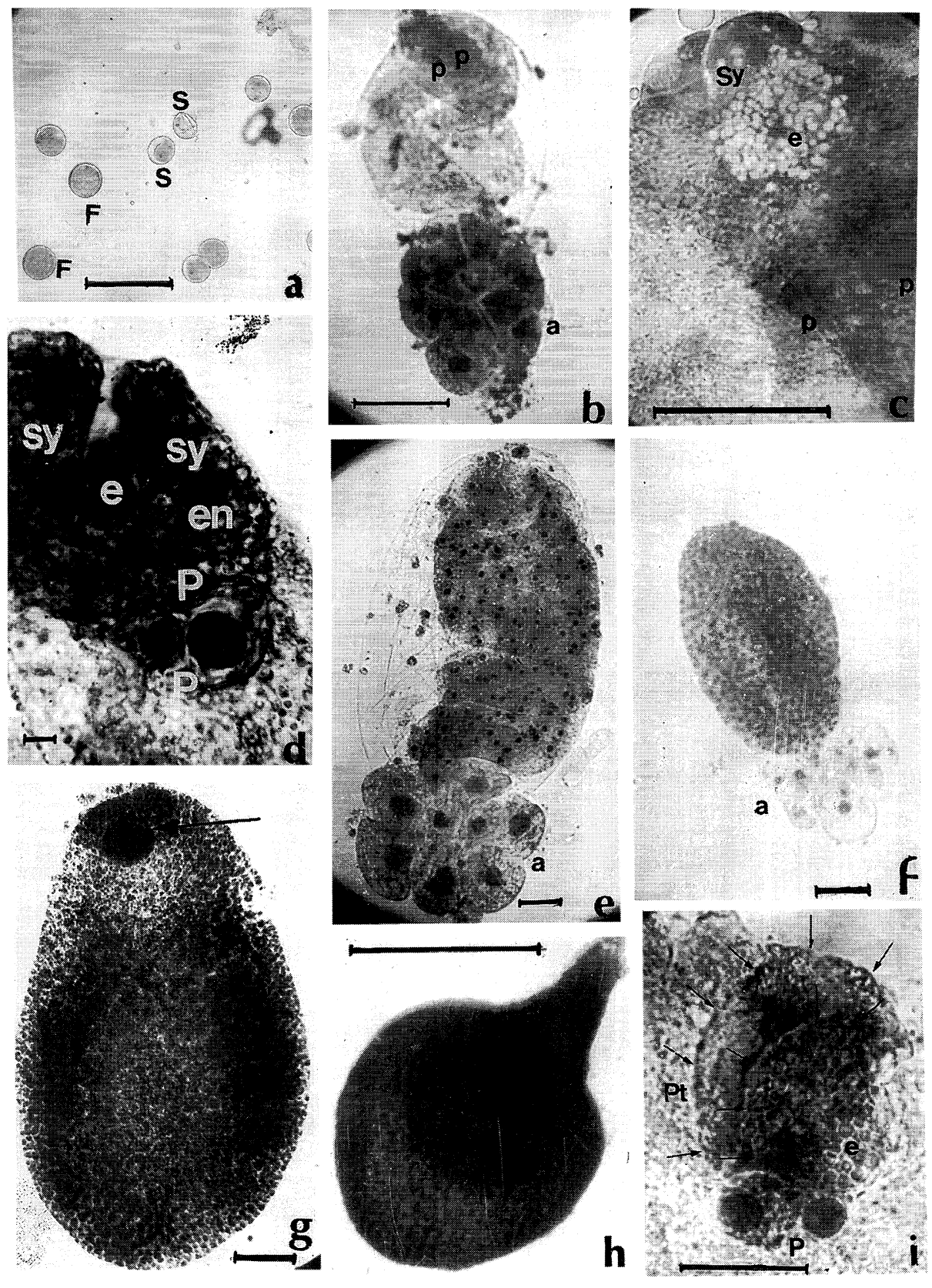

Fig. 2. Male and female gametophytes and post pollination developmental stages in the T3-6 interchange heterozygote of pearl millet. a) Pollen grains (male gametophytes), $\mathrm{F}=$ fertile, $\mathrm{S}=$ binucleate sterile (dp/df). b) Embryo sac (female gametophyte) from the ovule of a floret of an ear at ready to be pollinated stage (pre-pollination stage) $\mathrm{a}=$ antipodal cluster, $\mathrm{p}=$ polar nuclei. c, d) Micropylar end of the unfertilized embryo sac magnified, e=egg cell, Sy=synergid, $p=$ polar nuclei, en=egg nucleus. e) An embryo sac 24 hap with $\sim 64$ nucleate endosperm, a=antipodal cluster. f) An embryo sac at 48 hap with a $\sim 700$ celled endosperm $\mathrm{a}=$ degenerating antipodal cluster. g) An embryo sac 72 hap with a $\sim 2900$ celled endosperm and a globular embryo $(\rightarrow)$. h) An embryo from a 96 hap sample, i) Micropylar end of the embryo sac magnified showing pollen tube $(\mathrm{pt}, \rightarrow)$ adjacent to the egg cell $(\mathrm{e}), \mathrm{p}=$ polar nuclei. 
Table 1. Post pollination development in the T(3-6) interchange heterozygote of pearl millet

\begin{tabular}{cccc}
\hline \hline & $\begin{array}{c}\text { \% Unfertilized* } \\
\text { embryo sacs }\end{array}$ & Fertilized embryo sacs \\
\cline { 3 - 4 } & & \% with normal Dev. ${ }^{*}$ & \% with lag in Dev.* \\
\hline 24 & 30 & 70 & 18 \\
78 & 16 & 66 & 33 \\
96 & 17 & 50 & 30 \\
\hline
\end{tabular}

hap=hours after pollination. * Values calculated from observations in 250 ovules, all ovules had a structurally normal looking embryo sac.

Table 2. Rate of endosperm and embryo development in the T(3-6) interchange heterozygote of pearl millet

\begin{tabular}{|c|c|c|c|c|c|c|c|c|}
\hline \multirow{2}{*}{ hap } & \multirow{2}{*}{$\begin{array}{l}\text { Type } \\
\text { of dev. }\end{array}$} & \multicolumn{4}{|c|}{ Endosperm } & \multicolumn{3}{|c|}{ Embryo } \\
\hline & & $\begin{array}{c}\text { Mean No. of } \\
\text { nuclei }^{\S}\end{array}$ & $\begin{array}{c}\text { No. of cell } \\
\text { cycles }\end{array}$ & $\begin{array}{l}\text { Mean cycle } \\
\text { time (hr) }\end{array}$ & $\begin{array}{l}\text { Reported cycle } \\
\text { time* }(\mathrm{hr})\end{array}$ & $\begin{array}{c}\text { Mean No. of } \\
\text { cells }^{\S}\end{array}$ & $\begin{array}{c}\text { Reported } \\
\text { No. of cells* }\end{array}$ & $\begin{array}{c}\text { No. of cell } \\
\text { cycles }\end{array}$ \\
\hline 24 & Normal & $53.8 \pm 5$ & 6 & $4.1 \pm 0.3$ & $4.7 \pm 0.13$ & 2 & 2 & 1 \\
\hline \multirow[t]{2}{*}{48} & Normal & $695 \pm 77$ & 9 & $8.0 \pm 0.3$ & $4.6 \pm 0.00$ & $19 \pm 0.7$ & $17.2 \pm 0.9$ & 4 \\
\hline & Lag & $248 \pm 44$ & 8 & $12.1 \pm 0.43$ & - & $14 \pm 6$ & - & 4 \\
\hline \multirow[t]{2}{*}{72} & Normal & $2866 \pm 673$ & 11 & $12.0 \pm 0.66$ & $11.5 \pm 1.2$ & $61 \pm 2.8$ & $142.6 \pm 17$ & $* *$ \\
\hline & Lag & $540 \pm 36$ & 9 & $24.3 \pm 0.32$ & - & $23 \pm 4$ & - & $* *$ \\
\hline \multirow[t]{2}{*}{96} & Normal & $4921 \pm 463$ & $* *$ & $* *$ & $* *$ & $470 \pm 46$ & $279 \pm 32$ & $* *$ \\
\hline & Lag & $998 \pm 64$ & 10 & $24.5 \pm 0.46$ & - & $112 \pm 18$ & - & $* *$ \\
\hline
\end{tabular}

hap $=$ Hours after pollination. * Values estimated in sib crosses of the T(3-6) interchange homozygote by Krishna Rao and Aswani Kumari (1985). ** Values not calculated because synchrony in cell division is lost. ${ }^{\S}$ Calculated from 10 ovules.

ples of the interchange heterozygote. $16-30 \%$ of the embryo sacs remained unfertilized, $18-33 \%$ of the ovules showed a lag in post pollination development.

The unfertilized embryo sacs in the post pollination samples of the interchange heterozygote had a healthy appearance even at 96 hap. They did not degenerate up till this stage. No pollen tube was observed in most of these embryo sacs, only in two of them, a pollen tube was seen (Fig. 2i). In pearl millet, normally a pollen tube enters the embryo sac within $45 \mathrm{~min}$ to $1 \mathrm{hr}$ after pollination (Krishna Rao and Aswani Kumari 1981). Thus these embryo sacs failed to attract the pollen tubes and hence remained unfertilized.

The second kind of abnormality observed in the post pollination samples was a slower rate of development. In such ovules a lag in development was first noticeable in 48 hap sample, at this stage it was evident in the endosperm; the embryo, however, had the expected number of cells (Table 2). By 72 hap the development of the embryo also lagged behind, it had fewer number of cells (Table 2). At 96 hap the number of nuclei in the endosperm reached a stage at which cellularization usually takes place. But the endosperm in these ovules (failed to cellularize and) remained free nuclear.

The pollen fertility in the interchange heterozygote line ranged between $36-56 \%$ and seed set on open pollinated ears varied between $33-52 \%$.

\section{Discussion}

In the T3-6 interchange heterozygote of pearl millet, while breakdown in development of male gametophyte in the $\sim 50 \% \mathrm{dp} / \mathrm{df}$ microspores occurred midway after one mitosis, a normal looking embryo sac was found in all the ovules. However, post pollination, normal development was ob- 
served in only about half of them. The others with abnormalities are inferred to be dp/df. The two sexes thus differed in their ability to cope with the genetic lesion: the $\mathrm{dp} / \mathrm{df}$ microspores could progress only up to midway in male gemetophyte development while the $\mathrm{dp} / \mathrm{df}$ megaspores managed to complete normal female gametophyte development. This difference in the level of tolerance to the $\mathrm{dp} / \mathrm{df}$ condition between the two sexes could be due to the following reasons.

The microspore expresses its own genes rather than the phenotype of the parent (Walden and Greyson 1986). Intense gene activity has been observed in the pollen (Mascarenhas et al. 1986, Sari Gorla et al. 1986, Mulcahy 1987). Therefore, it is possible that microspores with dp/df chromosomal condition cannot develop normally. The development of the female gametophyte could however be directed by the maternal genome. Studies of the female gametophyte in the autotetraploids and a desynaptic mutant in pearl millet point towards this conclusion (Koduru and Krishna Rao 1978, Aswani Kumari 1981, Murthy 1982). Apparently, a supportive role for the maternal tissue is "normal". In both the autotetraploid and the desynaptic mutant, the percent pollen fertility correlated with percent seed set. In the autotetraploid pearl millet, while $\sim 20 \%$ microspores with aneuploid condition did not initiate male gametophyte development and appeared as sterile pollen (Koduru and Krishna Rao 1978), 95\% of the ovules possessed normal looking embryo sacs (Krishna Rao and Aswani Kumari 1980). In the monofactorially inherited desynaptic mutant of pearl millet (showing extreme desynapsis), only $8 \%$ of the pollen looked normal fertile (Murthy 1982), whereas $78-83 \%$ of the ovules had normal looking embryo sacs (Aswani Kumari 1981). Similar is the situation in the T3-6 interchange heterozygote of pearl millet described here.

The higher level of tolerance to chromosomal aberrations on the female side than in the male counterpart could also be due to the absence of competition from (mega-) spores with normal chromosome complement in the ovule (Sybenga 1992). There is only one megaspore mother cell in the ovule and when the interchange multivalent shows adjacent or indifferent orientation, all the resultant megaspores are $\mathrm{dp} / \mathrm{df}$. The $\mathrm{dp} / \mathrm{df}$ megaspores in the absence of competition from normal spores could have utilised the nutrients from the maternal tissue and developed into normal looking embryo sacs. The dp/df microspores on the other hand have an approximately equal number of microspores with normal chromosome complement to compete with (Janse 1987). "Functionally less competent $\mathrm{dp} / \mathrm{df}$ or aneuploid male gametophytes" in some chromosomal interchanges and trisomics of maize and rye were found to succeed in fertilization when, artificially, the chromosomally balanced or euploid fertile pollen was excluded (Walden and Greyson 1986, Janse 1987, Niizeki and Saito 1988). In Arabidopsis thalliana the dp/df megaspores of a translocation (involving chromosomes 2 and 5) heterozygote failed to develop into embryo sacs. In the dp/df megaspores either a cell division was not initiated or development stopped after one division. They remained uni- or bi-nucleate (Ray et al. 1997). Whether they represent the two kinds of dp/df spores is not mentioned by Ray et al. (1997). The dp/df region in these spores must have had genes controlling cell division/development.

The two kinds of dp/df spores could not be distinguished on the male side in the T3-6 interchange heterozygote of pearl millet. Both kinds of microspores degenerated at binucleate stage. On the female side, in the dp/df products, functional incompetence was visible at two different-gametic and zygotic (embryonic) stages. Some embryo sacs failed to get fertilized (gametic lethality), some succeeded in fertilization but the embryo degenerated (apparently) due to the $\mathrm{dp} / \mathrm{df}$ condition (zygotic lethality). These two kinds might represent the embryo sacs with the different $\mathrm{dp} / \mathrm{df}$ conditions. Differences in the two kinds dp/df products have been reported on the male side in some interchanges of maize and Allium triquetrum (Burnham 1962, Rickards 1983, Walden and Greyson 1986). We have found such differences (Table 1) on the female side in the T3-6 interchange heterozygote of pearl millet.

In the dp/df embryo sacs that failed to attract the pollen tube (and therefore not succeed in fertilization) the genetic lesion could have affected their ability to elicit a chemotropic signal which di- 
rects the pollen tube into them. Control of pollen tube growth by the embryo sacs was first reported in avocado (Sedgley 1978). In an interchange heterozygote of Arabidopsis thalliana the ovules with no embryo sac did not have a pollen tube (Ray et al. 1997). Ray et al. (1997) concluded that the pollen tube is guided by the female gametophyte. The synergids are assumed to synthesise chemotropic substances (Went and Willemse 1986). A calcium gradient formed by synergids has also been implicated in the chemotropic signal (Chaubal and Reger 1990, Reger et al. 1992). A considerable number of the normal looking embryo sacs in the desynaptic mutant and autotetraploid of pearl millet (aneuploid for different chromosomes) also failed to get fertilized when pollinated (Aswani Kumari 1981, Krishna Rao and Aswani Kumari 1981). Thus the genetic elements governing the chemotropic function of the embryo sac may be distributed on several chromosomes.

The $\mathrm{dp} / \mathrm{df}$ embryo sacs that succeeded in fertilization did not achieve a normal rate of development. The triploid endosperm was the first to be affected, its nuclei have two sets of $\mathrm{dp} / \mathrm{df}$ chromosomes. The embryo coped for a longer while; its nuclei have one set of $\mathrm{dp} / \mathrm{df}$ chromosomes. Eventually both degenerated. Degeneration of the embryo during early stages of seed development has also been observed in some ovules in the desynaptic mutant and autotetraploid of pearl millet (Aswani Kumari 1981, Krishna Rao and Aswani Kumai 1980). The early development of the embryo is believed to be directed by the gene activity in the egg (Raghavan and Sharma 1995). Intense gene activity as evidenced by presence of labelled mRNA has been reported in the egg cell (Raghavan 1990, Bimal et al. 1994, Bimal and Willemse 1996). Thus the chromosomally unbalanced egg could not sustain normal development of the zygote. At present there is little data available about the regulation of ovule and embryo sac development and the temporal and spatial patterns of gene expression in the embryo sac (Reiser and Fisher 1984). For example the extent to which the genetic programmes overlap between the male and female gametophytes on one hand and between the female gametophyte and sporophyte on the other is not known. Also unknown is the time when actually the gametophyte specific genes are expressed during the development of the female gametophyte (Reiser and Fisher 1984). The molecular genetic approaches required to address some of these questions could be done using this easy technique of isolation of embryo sacs and the cytogenetic mutants as the one described here.

\section{Acknowledgements}

We thank the special assistance programme for cytogenetics to the Botany Department by the University Grants Commission, New Delhi for financial assistance. Lakshmi, T. V. R. and Aruna Lakshmi, K. thank the Council for Scientific and Industrial Research, New Delhi for Research Associateships.

\section{References}

Adiseshiah, K. 1995. Male and female gametophyte development in the duplicate deficient products of two interchange heterozygotes and a $\mathrm{GA}_{3}$ induced sterile inbred line of pearl millet (Pennisetum glaucum (L.) R. Br.). M. Phil. Dessert. Andhra Univ. India.

Aswani Kumari, K. 1981. Rate of cell development during early seed formation in pearl millet, Pennisetum americanum (L.) Leeke. Ph.D. Dessert. Andhra Univ. India.

Bimal, R., Schel, J. H. N., Tavoletli, S. and Willemse, M. T. M. 1994. Localization of Poly(A) ${ }^{+}$containing RNA during female gametophyte development in Medicago sativa, and the diploid mutant Medicago falcata using digoxigenin Oligo dT probes. Sex. Pl. Repr. 8: 18-26.

Bimal, R. and Willemse, M. T. M. 1996. Distribution of poly (A) ${ }^{+}$containing RNA during female gametophyte development in Gasteria Verrucosa (Mill.) H. Duval. Phytomorphology 46: 9-17.

Burnham, C. R. 1962. Interchanges In: "Discussions in Cytogenetics". pp. 66-116. Burgees Publ. Co. Minneapolis, Minnesota, USA

Chaubal, R. and Reger, B. J. 1990. Relatively high calcium is localized in synergid cells of wheat ovaries. Sex. Pl. Repr. 4: 
98-102.

Janes, J. 1987. Certation between euploid and aneuploid pollen grains from a tertiary trisomic of rye, Secale cereale L. Genome 29: 353-356.

Khush, G. S. 1973. Cytogenetics of Aneuploids. Academic Press, New York.

Koduru, P. R. K. 1979. Metaphase I centromere co-orientation in interchange heterozygotes of pearl millet. Genet. Res. 34: 69-76.

—and Krishna Rao, M. 1978. Chromosome pairing and desynapsis in spontaneous autoploids of Pennisetum americanum. Cytologia 43: 445-452.

- 1984. Cytogenetics of a semidwarf phenotype in pearl millet, Pennisetum americanum (L.) Leeke. Canadian J. Genet. Cytol. 26: 272-278.

Krishna Rao, M. and Aswani Kumari, K. 1980. Effect of autopolyploidy on the rate of cell development during early seed formation of pearl millet. J. Genet. Cytol. 15: 134-139.

- 1981. Cell development in the young seed of two inbred lines of pearl millet, Pennisetum americanum (L.) Leeke. Australian J. Bot. 29: 617-624.

- 1985. Cell development during early embryo and endosperm development in eight lines of pearl millet, Pennisetum americanum (L.) Leeke. In: "Experimental Manipulation of Ovular Tissues" (G. P. Chapman, S. H. Mantell and R. W. Daniels (Eds.)), pp. 151-153. Ptimans, London.

Mascarenhas, J. P., Stinson, J. S., Willig, R. P. and Enlicope, M. 1986. Genes and their expression in the male gametophyte of flowering plants. In "Biotechnology and Ecology of Pollen" (D. L. Mulcahy, G. B. Mulcahy and E. Ottaviano (Eds.)), pp. 39-44, Springer-Verlag, Berlin.

Mulcahy, D. L. 1987. Gametophytic gene expression. In: "A Genetic Approach to Plant Biochemistry" (A. D. Bionstein and P. J. King (Eds.)), pp. 247-258, Narosa Publ. House, New Delhi, India.

Murthy, T. G. K. 1982. Cytogenetics of six spontaneous meiotic mutants of pearl millet, Pennisetum amerianum (L.) Leeke. Ph.D. Dessert. Andhra Univ. India.

Niizeki, M. and Saito, K. 1986. Increasing the transmission rate of the extra-chromosome in a trisomic Nicotiana sulvestris line by modifying the means of pollination. Theor. Appl. Genet. 76: 891-896.

Raghavan, V. 1990. Spatial Distribution of mRNA during prefertilization ovule development in Capsella hursa pastoris. Sex. Pl. Repr. 3: 170-178.

Raghavan, V. and Sharma, K. K. 1995. Zygotic embryogenesis in gymnosperms and angiosperms. In: "In Vitro Embryogenesis in Plants; Current Plant Science and Biotechnology Agriculture” (T. A. Thorpe (Ed.)), pp. 73-115, Kluwer Publ., Netherlands.

Ray, S., Park, S. S. and Ray, A. 1997. Pollen tube guidance by the female gametophyte. Development 124: 2489-2498.

Reger, B. J., Chaubal, R. and Pressy, R. 1992. Chemotropic responses by pearl millet pollen tubes. Sex. P1. Repr. 5: 47-56.

Reiser, L. and Fisher, R. L. 1994. The ovule and the embryo sac. Plant Cell 5: 1291-1301.

Rickards, G. K. 1983. Orientation behaviour of chromosome multiples of interchange (reciprocal translocation) heterozygotes. Annu. Rev. Genet. 17: 443-498.

Sari Gorla, M., Frova, C. and Redaelli, E. 1986. Extent of gene expression at the gametophytic phase in maize. In: "Biotechnology and Ecology of Pollen" (D. L. Mulcahy, G. B. Mulcahy and F. Ottaviano (Eds.)), pp. 27-32. Springer-Verlag, Berlin.

Sedgley, M. 1976. Control by the embryo sac over pollen tube growth in the style of the avocado (Persea americana Mill.). New Phytol. 77: 149-152.

Sybenga, J. 1992. Karyotype variants A: chromosome structural variants. In: "Cytogenetics in Plant Breeding" (R. Frankel, M. Grossman and P. Maliga (Eds.)), Monographs on Theoretical and Applied Genetics 17: pp. 101-139, SpringerVerlag, Berlin.

Uma Devi, K., Narasinga Rao, P. S. R. L. and Krishna Rao, M. 1996. Linkage of semidwarf phenotype to interchange homozygosity in pearl millet. J. Hered. 87: 170-172.

Walden, D. B. and Greyson, R. I. 1986. Maize pollen research: preliminary reports from two projects investigating gametic selection. In: "Biotechnology and Ecology of Pollen" (D. L. Mulcahy, G. B. Mulcahy and E. Ottaviano (Eds.)), pp. 139-145, Springer-Verlag, Berlin.

Went, V. J. L. and Willemse, M. T. M. 1984. Fertilization. In: "Embryology of Angiosperms" (B. M. Johri (Ed.)), pp. 273-317, Springer-Verlag, Berlin. 